\title{
Ocorrência de Casos de Intoxicação por Plantas Ornamentais Tóxicas no Estado de Mato Grosso do Sul
}

\section{Occurrence of Cases of Poisoning by Toxic Ornamental Plants in the State of Mato Grosso do Sul}

\author{
Carolina Cereser Fracaro ; Vera Lucia Ramos Bononia; Denise Renata Pedrinho*b; José Antônio Maior Bono ${ }^{\mathrm{b}}$; \\ Rosemary Matias; Giovana Coutinho Zulin Nascimento ${ }^{\mathrm{a}}$; Cinthia dos Santos Lopes
}

\begin{abstract}
aUniversidade Anhanguera-Uniderp, Programa de Pós-Graduação Stricto Sensu em Meio Ambiente e Desenvolvimento Regional. MS, Brasil. ${ }^{b}$ Universidade Anhanguera-Uniderp, Programa de Pós-Graduação Stricto Sensu em Produção e Gestão Agroindustrial. MS, Brasil.

*E-mail: denise.pedrinho@anhanguera.com
\end{abstract}

\begin{abstract}
Resumo
Os espaços verdes urbanos são um elemento-chave para a interação entre os cidadãos e o meio ambiente, além de promover a saúde humana. Porém, muitas plantas ornamentais presentes nestes espaços podem ser tóxicas, podendo ocasionar acidentes, principalmente em crianças. O objetivo deste trabalho foi realizar um levantamento das intoxicações por plantas ornamentais tóxicas ocorridas no período de 2005 a 2015 no Estado de Mato Grosso do Sul. Dados do Centro Integrado de Vigilância Toxicológica (CIVITOX) registraram 214 casos de intoxicações por plantas ornamentais nesse período, sendo que a maioria dos casos está concentrado em Campo Grande, porém a região de Rio Verde e Coxim apresentaram os maiores índices de intoxicação por 5.000 habitantes. Pessoas de todas as idades são intoxicadas com plantas, mas as crianças são a maioria das vítimas $(84 \%)$ e os dados mostraram haver correlação entre as variáveis mês, população e idade de intoxicação. A família Aracea foi predominante em números de casos de intoxicação, com 150 casos, dos quais 135 foram de intoxicações pela espécie conhecida como comigo-ninguém-pode (Dieffenbachia picta Schott). Cerca de cinquenta casos de intoxicações por plantas ornamentais tóxicas com faixa etária mais elevada foi registrada nas regiões centro-norte e centro-sul de Mato Grosso do Sul. Os resultados sugerem que a escolha de plantas ornamentais para compor as áreas verdes dos municípios deve ser baseada com critérios que possam minimizar os riscos para a população que utilizam esses espaços para o lazer.
\end{abstract}

Palavras-chave: Áreas Verdes. Intoxicação Humana. Planejamento Urbano. Centro Integrado de Vigilância Toxicológica. Dieffenbachia picta.

\begin{abstract}
The green areas of cities have the function of promoting interaction between citizens and the environment, in addition to promoting human health. The plants used to compose these areas, which are ornamental, can be toxic and cause accidents, especially in children. The objective of this work was to conduct a survey of poisoning by toxic ornamental plants that occurred in the period from 2005 to 2015 in the State of Mato Grosso do Sul, using the integrated toxicological surveillance center (CIVITOX). A total of 214 cases of poisoning by ornamental plants were recorded during this period, and most cases went to the city of Campo Grande. The region of Rio Verde and Coxim has rathe highest rates of intoxication, when related to the number of intoxications by the number of habitants. The records show people of various age groups with intoxicated plants, but children are the majority of victims (84\%) and in the data analysis, there was a correlation between the month, population and age of intoxication. The records show people of various age group with intoxicated plants, but children are the majority of victims (84\%) and in the analysis of the data, there was a correlation between the month, population and age of intoxication. The Aracea family was predominant in numbers of cases of intoxication, with 150 cases, of which 135 were poisonings by the species with me-nobody-can (Dieffenbachia picta Schott). The highest age group, with more than 50 cases of poisoning, had a predominance of occurrence in the northcentral and central-south regions of Mato Grosso do Sul.The results suggest that the choice of ornamental plants to compose the green areas of the municipalities should be based on criteria that can minimize the risks to the population that use these spaces for leisure.
\end{abstract}

Keywords: Green Areas. Human Poisoning. Urban Planning. Integrated Toxicological Surveillance Center, Dieffenbachia Picta.

\section{Introdução}

A relação da humanidade com agentes tóxicos vem desde a antiguidade. No entanto, foi apenas há 50 anos que surgiram as primeiras entidades ligadas ao controle das intoxicações, tendo início na Europa, mais precisamente na Bulgária e Inglaterra e nos Estados Unidos (BURDA, 1997).

Já no Brasil, foi ao longo da década de 1970, por iniciativas particulares e desvinculadas de qualquer política pública formal, que começaram a surgir os primeiros Centros de Informação Toxicológica. A demanda por atendimento às intoxicações e a necessidade de prestar um atendimento profissional de qualidade definiram os locais nos quais esses Centros foram implantados. O primeiro Centro surgiu na cidade de São Paulo, na década de 1970 e foi considerada semente da toxicologia médica no Brasil (AZEVEDO, 2010).

Na década de 1980 surgiu o primeiro Centro de Informação Toxicológica (CIT) na região Centro-oeste. Localizado em Campo Grande, capital de Mato Grosso do Sul, este ficou inicialmente conhecido por CIT/MS. Em 2002, por ação do governo estadual ele passou a funcionar no Hospital Regional Estadual.

Os Centros de informações toxicológicas procuram 
registrar todas as ocorrências de envenenamentos e entre elas as causadas por plantas toxicas. Definem-se como plantas tóxicas todo vegetal que, ingerido ou em contato com pele, mucosas e olhos do homem ou animais, em condições naturais, é capaz de causar danos que refletem na saúde e vitalidade do organismo desses seres (TOKARNIA et al., 2000; ANDRADE FILHO et al., 2013).

Segundo dados da FIOCRUZ (2016), em 2013 foram registrados 441 casos de intoxicação por plantas no Brasil. A região Sudeste concentra o maior número desses casos (259), seguida pela região centro-oeste, com 129 casos e nordeste com 35 registros. A região centro-oeste, com seu total de 129 casos de intoxicações por plantas, tem Brasília com o maior número de registros (92), seguida por Campo Grande, com 33 casos. Um dado preocupante é a faixa etária mais acometida por essas intoxicações, pois do total de 441 registros de intoxicações por plantas, 307 ocorreram com crianças de 0 a 9 anos de idade.

As intoxicações por plantas em sua grande maioria decorrem de ingestões acidentais e principalmente, por desconhecimento da população, sobre os perigos que determinadas espécies utilizadas como ornamentais podem causar à saúde humana.

Vasconcelos et al. (2009) revelam que a cada dez casos de intoxicação por plantas, seis acontecem em crianças menores de nove anos de idade, devido à presença de plantas tóxicas em espaços públicos, inclusive nas escolas, sendo assim as crianças, o grupo mais suscetíveis à agressividade dos princípios ativos tóxicos existentes nessas espécies.

O conhecimento acerca das plantas e das suas propriedades tóxicas, principalmente daqueles presentes no cotidiano das pessoas, é o principal caminho contra acidentes (SILVA et al., 2015). Tendo em vista que as plantas ornamentais tóxicas são causas frequentes de intoxicações e constitui importante problema de saúde pública, o objetivo desse estudo foi realizar um levantamento junto ao Centro Integrado de Vigilância Toxicológica (CIVITOX), sobre os casos de intoxicação por plantas ornamentais tóxicas no estado de Mato Grosso do Sul no período de 2005 a 2015.

\section{Material e Métodos}

A pesquisa foi desenvolvida no município de Campo Grande, capital de Mato Grosso do Sul. Os dados foram obtidos no centro de informação e assistência toxicológica de Mato Grosso do Sul, CIVITOX (Centro Integrado de Vigilância Toxicológica), localizado em Campo Grande, MS.

Foram contemplados os dados referentes aos casos de intoxicação por plantas ornamentais de todo Estado de Mato Grosso do Sul, além da capital Campo Grande. O CIVITOX fornece informações toxicológicas aos profissionais da saúde e à população em geral, atende pacientes intoxicados em regime de urgência, se vinculado a instituições hospitalares, capta informações epidemiológicas confiáveis, gera estudos epidemiológicos para embasar políticas públicas, confirma intoxicação com base em princípios básicos e protocolos de tratamento, realiza busca ativa de casos, divulga alertas epidemiológicos, acompanha ativamente os casos notificados e atendidos, atua na área da prevenção, fornece suporte laboratorial, opera na produção cientifica e na formação de pessoal (AZEVEDO, 2010).

O funcionamento é em regime permanente, 24 horas, com atendimento via telefone aos profissionais e à população que buscam informações para os casos de intoxicações, abrangendo atendimentos de toda extensão territorial de Mato Grosso do Sul. No CIVITOX, os registros de casos de intoxicação são realizados por meio do preenchimento de um formulário que contempla dados tais como: hora e data da ocorrência da intoxicação, nome, idade e data de nascimento do indivíduo intoxicado, endereço, cidade onde ocorreu a intoxicação, agente tóxico que causou a intoxicação, entre outras informações.

Os formulários são organizados em arquivos separados por ano de ocorrência, subdivididos em meses, de acordo com as classes de agentes tóxicos, tais como: medicamentos, agrotóxicos de uso agrícola, agrotóxicos de uso doméstico, produtos veterinários, raticidas, domissanitários, cosméticos, produtos químicos industriais, metais, drogas de abuso, plantas, alimentos, animais peçonhentos/serpentes, animais peçonhentos/aranhas, outros animais peçonhentos/venenosos, animais não-peçonhentos, desconhecidos e outros.

Para essa pesquisa foram levantados os casos de intoxicação humana por plantas ornamentais tóxicas em Mato Grosso do Sul, no período entre 2005 a 2015. A princípio a busca foi aos formulários com registros de intoxicações por plantas, posteriormente foram separados os casos de intoxicações por plantas ornamentais tóxicas, de acordo com bibliografia especializada, Matos et al. (2011), Livro de Plantas Tóxicas; Winters (2013), Apostila de Plantas Ornamentais Tóxicas; Lorenzi (2013), Plantas para Jardim no Brasil.

Dentre as informações contidas nos formulários, utilizaram-se as variáveis agente tóxico (espécies ornamentais causadoras de intoxicações); município (gerando dados referentes à distribuição do número de casos de intoxicação por plantas ornamentais tóxicas no Estado e o índice de intoxicação por 5000 habitantes) e faixa etária.

Os dados levantados foram submetidos à análise estatística, univariada através de correlação simples, análise multivariada com determinação dos componentes principais e análise espacial através da geoestatística. Para análise de correlação de componentes principais utilizou-se do programa SAS (SAS, 2002) nos procedimentos Proc Corr e Proc princomp e na análise da geoestatística e elaboração dos mapas o programa Surfer (SURFER, 2000).

\section{Resultados e Discussão}

Foram registrados pelo CIVITOX 214 casos de intoxicação humana por plantas ornamentais tóxicas entre 
os anos de 2005 e 2015 no Estado de Mato Grosso do Sul, com 23 diferentes espécies causadoras de tais ocorrências, pertencentes a 14 famílias (Quadro 1). Com o maior número de espécie está a família Araceae (5 espécies) seguida das famílias Euphorbiaceae e Solanaceae (3 espécies), as demais famílias com uma espécie.

Quadro 1 - Espécies ornamentais tóxicas registradas pelo CIVITOX como causadoras de intoxicação humana em Mato Grosso do Sul no período de 2005 a 2015

\begin{tabular}{|c|c|c|c|}
\hline Família & Nome Científico & Nome Popular & $\mathrm{N}^{\mathrm{o}}$ de Casos de Int. \\
\hline \multirow{5}{*}{ Araceae } & Anthurium spp. & Antúrio & 03 \\
\hline & Monstera deliciosa $\mathrm{L}$. & Costela-de-adão & 01 \\
\hline & Dieffenbachia picta Schott. & Comigo-ninguém-pode & 135 \\
\hline & Zantedeschia aethiopica $\mathrm{L}$. & Copo-de-leite & 07 \\
\hline & Zamioculcas zamiifolia & Zamioculca & 04 \\
\hline Amaranthaceae & Celosia cristata $\mathrm{L}$. & Crista-de-galo & 01 \\
\hline Apocynaceae & Nerium oleander L. & Espirradeira & 03 \\
\hline Araliaceae & Hedera helix $\mathrm{L}$. & Hera & 01 \\
\hline Asteraceae & Senecio spp. & Flor-das-almas & 01 \\
\hline Cactaceae & Cactus spp. & Cacto & 01 \\
\hline Convolvulaceae & Ipomoea quamoclit L. & Esqueleto & 01 \\
\hline \multirow{3}{*}{ Euphorbiaceae } & Euphorbia milii Des Moulins. & Coroa-de-cristo & 25 \\
\hline & Euphorbia heterophylla L. & Leiteiro & 01 \\
\hline & Euphorbia tirucalli $\mathrm{L}$. & Avelós & 15 \\
\hline Leguminosae & Senna macranthera DC. Ex collad. & Aleluia & 01 \\
\hline Nyctaginaceae & Mirabilis jalapa L. & Maravilha & 01 \\
\hline Pteridaceae & Adiantum capillus-veneris & Avenca & 01 \\
\hline \multirow{3}{*}{ Solanaceae } & Atropa belladonna L. & Beladona & 04 \\
\hline & Cestrum nocturnum L. & Dama-da-noite & 02 \\
\hline & Datura suaveolens Willd. & Saia-branca & 03 \\
\hline \multirow{2}{*}{ Verbenaceae } & Duranta repens L. & Pingo-de-ouro & 01 \\
\hline & Lantana câmara L. & Cambará & 01 \\
\hline Urticaceae & Caliisia repens & Dinheiro-em-penca & 01 \\
\hline
\end{tabular}

$\mathrm{N}^{\circ}$ de Casos Int.= Número de Casos de Intoxicações.

Fonte: Centro Integrado de Vigilância Toxicológica (CIVITOX).

Observa-se que a família Araceae possui o maior número de casos (150), com destaque a planta ornamental comigo-ninguém-pode (Dieffenbachia picta Schott) com 135 ocorrências, seguida pela coroa-de-cristo (Euphorbia milii Des Moulins) e avelós (Euphorbia tirucalli L) com 25 e 15 casos registrados, respectivamente, ambas da família Euphorbiaceae com um total de 41 casos para as três espécies desta família.

A maioria das intoxicações por plantas ocorre por ingestão acidental, devido ao desconhecimento das populações sobre os perigos que algumas espécies ornamentais podem causar (SILVA et al., 2015). Segundo Fernandes et al. (2017), os acidentes com plantas representam a quarta principal causa de intoxicação no Brasil e têm sido um dos problemas para a saúde pública ao longo dos anos, especialmente crianças.

A Dieffenbachia picta é uma planta muito comum e abundante em todo o país, suas características botânicas (folhas brilhosas e grandes) chamam a atenção das pessoas e crianças que acabam manuseando ou mesmo ingerindo e se intoxicando, inclusive crianças (MENDIETA et al., 2014).

Caracterizada como uma das plantas que mais ocasionam intoxicações, sua ação tóxica é conhecida há muito tempo. A planta apresenta ráfides de oxalato de cálcio em formato de agulhas nas suas folhas e hastes, quando ingerida pode acarretar edema na língua, salivação, ulcera, diarreia e vômitos. O contato com os olhos causa dor, inchaço e lacrimejamento (CUMPSTON et al., 2003; SILVA; USHIROBIRA, 2010).

Euphorbia milii Des Moul. é uma planta arbustiva, perene, ramificada e com numerosos espinhos. Muito utilizada em paisagismo, como cerca viva, pois apresenta rusticidade e adaptabilidade a diferentes locais. As intoxicações com essa planta comumente acontecem no momento da poda.

As partes vegetais, com grande quantidade de látex, têm princípios tóxicos, os diterpenos, denominados miliaminas, responsáveis pela ação irritante quando em contato com a pele e mucosas, podendo causar edema, queimação e coceira, além de provocar irritação e lacrimejamento quando em atinge os olhos (UEMURA; HIRATA, 2001).

Diversas outras espécies foram registradas nesse estudo, como causadoras de intoxicações humanas, porém em menor número de ocorrência, como Euphorbia tirucalli, conhecida como aveloz, árvore do lápis, cachorro pelado, cega-olho, dedo do diabo, espinho italiano, entre outros. É uma espécie nativa da África, que chegou ao Brasil em 1892 inicialmente como ornamental. No entanto, esta planta é considerada altamente tóxica, pois possui fitoquímicos como compostos terpênicos (MATOS, 2000; DUONG et al., 2019). Ainda segundo os autores, as plantas do gênero Euphorbia são reconhecidas por 
suas inflorescências e pelo látex de coloração esbranquiçada que em contato com a pele tem ação irritante e caustica, e em contato com olhos pode determinar o desenvolvimento de conjuntivites, queratites e uveítes ou mesmo levar à cegueira. O látex exerce propriedades pesticidas contra várias pragas, incluindo mosquitos, informações estas demonstram que a toxicidade está relacionada com o látex, cuidados devem ser tomados com uso da planta como ornamental.

Resultados semelhantes aos encontrados nesse estudo foram descritos por Garcia e Baltar (2007), que analisando casos de intoxicações por plantas em Londrina, Paraná, registraram cinco espécies, entre elas, Dieffenbachia picta, Zantedeschia aethiopica, Euphorbia milii, Jatropha curcas e Ricinus communis.

De acordo com Dominato (2012), Dieffenbachia picta e Euphorbia milii foram as protagonistas dos casos de intoxicação por planta registrados em uma unidade básica de saúde (UBS) de Presidente Prudente, São Paulo.

No período em que esse estudo foi realizado, nota-se nos registros da CIVITOX, que houve uma grande variedade de espécies ornamentais causadoras de intoxicações, entre elas: copo-de-leite (Zantedeschia aethiopica), zamioculca (Zamioculcas zamiifolia), beladona (Atropa belladonna), antúrio (Anthurium andraenum), espirradeira (Nerium oleander) e saia-branca (Datura suaveolens).

Cabe ressaltar, a importância do conhecimento a respeito da toxicidade de plantas ornamentais comumente utilizadas em residências, praças e parques, pois o efeito tóxico pode conduzir a serias consequências (SILVA et al., 2015).

Quanto ao número de casos de intoxicação humana por plantas ornamentais registrados nas diferentes regiões em Mato Grosso do Sul, estão pontuados os municípios nos quais houve registro no CIVITOX de intoxicação por planta ornamental tóxica no período estudado, sendo os que estão nomeados os principais de cada região, as demais sedes do município com números de casos de intoxicações por plantas ornamentais estão marcados com um ponto vermelho (Figura 1). A região com maior número de casos foi a capital, Campo Grande e os municípios próximos, entre 45 a 135.

Figura 1 - Número de casos de intoxicação humana por plantas ornamentais, registrados do CIVITOX, por região no Estado do Mato Grosso do Sul entre 2005 e 2015

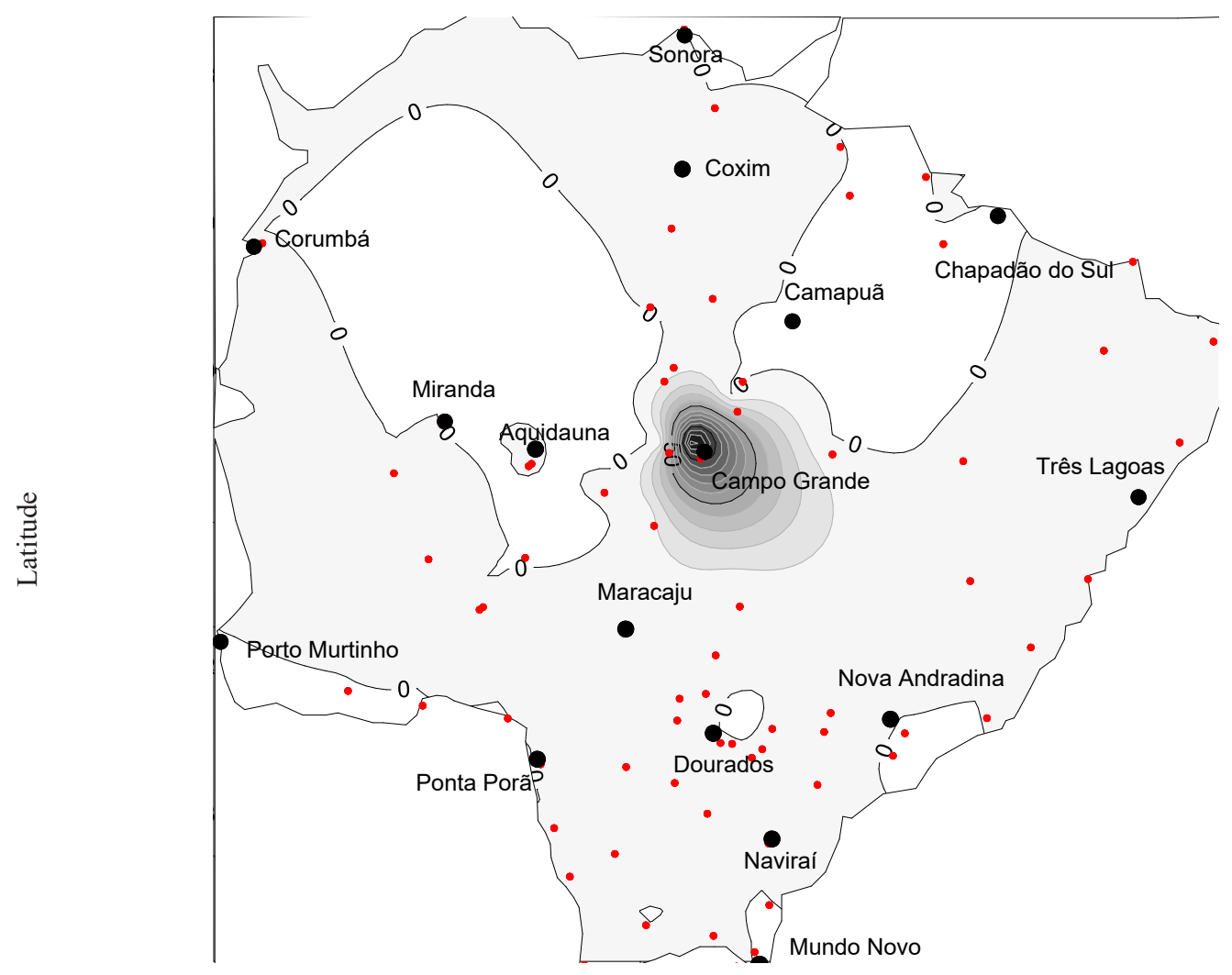

*Pontos vermelhos indicam a localização da sede dos Municípios

Longitude

Fonte: Centro Integrado de Vigilância Toxicológica (CIVITOX).

Isso pode ser justificado pelo acesso facilitado da população ao CIVITOX e a instituições de saúde de Campo Grande, como unidades básicas e hospitais, o que facilita o repasse desses casos ao Centro de Vigilância Toxicológica.

Já as pessoas que se intoxicam em municípios distantes da capital, estas não se deslocam até ela para receber atendimento 
médico, sendo feito na própria cidade e em alguns casos, essa ocorrência pode não ser repassada ao CIVITOX, o que torna as estatísticas incompletas, pois de acordo com Azevedo (2010), as estatísticas divulgadas pelo FIOCRUZ/CICT/SINITOX não contemplam a totalidade dos casos de intoxicações verificadas no Brasil, pois há insuficiência no número de Centros para abrangência de toda a extensão territorial no Estado e no Brasil.

Os índices de intoxicação por plantas ornamentais tóxicas entre os anos de 2005 e 2015 em Mato Grosso do Sul, encontram-se na Figura 2.

Figura 2 - Índice de intoxicação por plantas ornamentais no Estado do Mato Grosso do Sul (casos para cada 5000 habitantes), no período de 2005 a 2015 (CIVITOX)

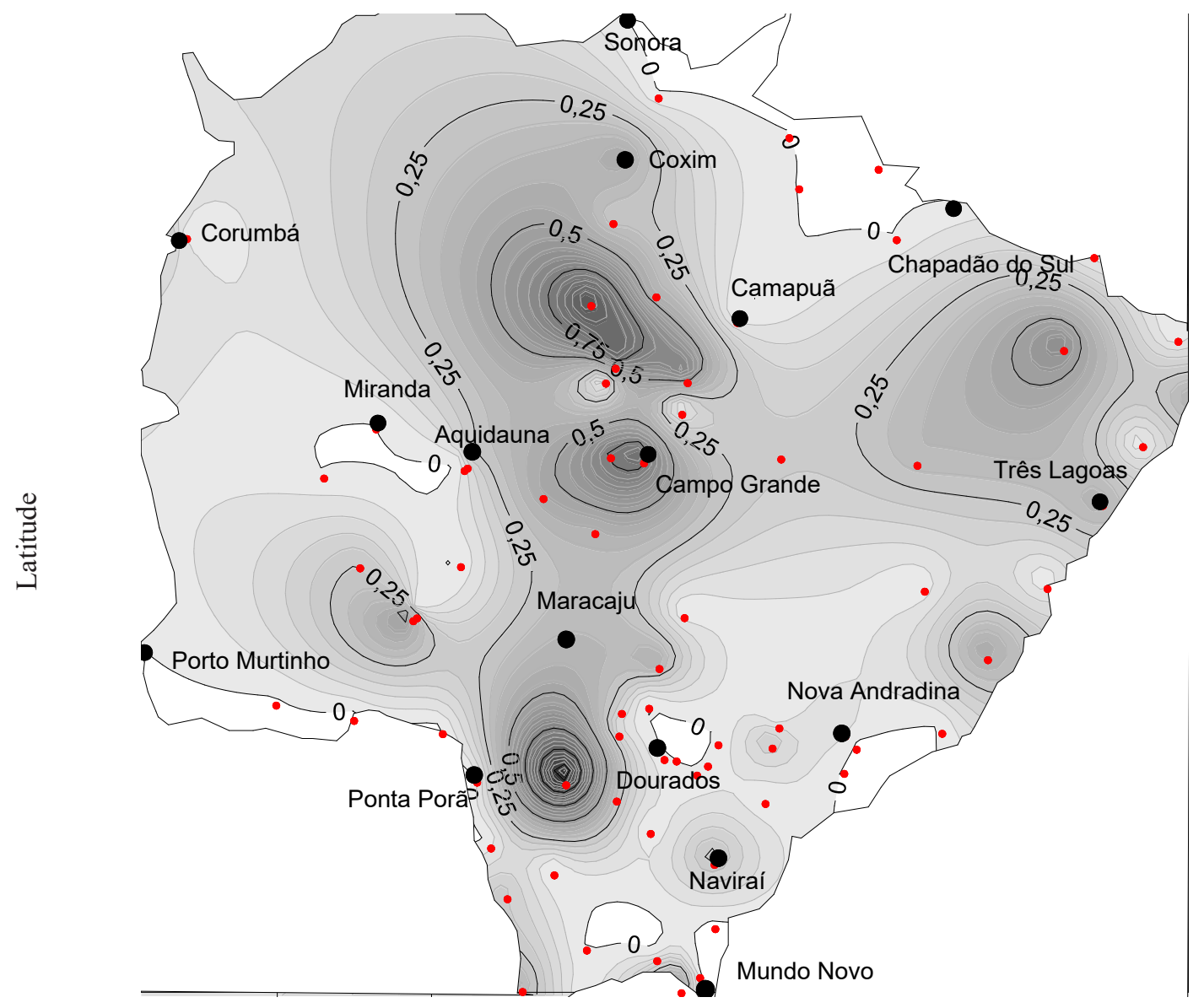

*Pontos vermelhos indicam a localização da sede dos Municípios Fonte. Centro Integrado de Vigilância Toxicológica (CIVITOX

Esse índice relaciona o número de casos registrados no município a cada 5.000 habitantes. Definiu-se 5.000, por se tratar do número aproximado de habitantes da cidade com menor população, a qual foi notificada com ocorrência de intoxicações, sendo este município Rio Negro, com estimativa de 4.910 habitantes (IBGE, 2010). Os pontos em vermelho caracterizam as demais sedes dos municípios com registros no CIVITOX.

O maior índice, 0,85 , é observado ao norte de Mato Grosso do Sul, abrangendo a região de Rio Verde de Mato Grosso e Coxim. A capital do Estado, Campo Grande, apresenta índice de 0,6, em função da grande quantidade de moradores no município, também registrado em municípios ao sul do Estado, na região de Dourados.
Longitude

Martins e Geron (2014) apontam que campanhas educativas e de prevenção junto à comunidade em geral, podem provocar mudanças de atitudes e diminuir os acidentes envolvendo intoxicações por plantas tóxicas. Para minimizar os riscos de acidentes, Biondi et al. (2008) sugerem a explanação através de placas informativas em praças, parques, escolas, entre outros, sobre as características das plantas tóxicas e os danos que podem causar.

$\mathrm{Na}$ Figura 3, são apresentados os dados referentes a intoxicações humanas, considerando a região de domicílio e a faixa etária registradas no CIVITOX entre 2005 e 2015. Os pontos em vermelho referem-se as sedes de municípios próximos as cidades pontuadas no mapa, com casos de intoxicações, registradas pelo CIVITOX. 
Figura 3 - Idade (anos) das pessoas intoxicadas por plantas ornamentais no Estado do Mato Grosso do Sul, no período de 2005 a 2015 (CIVITOX)

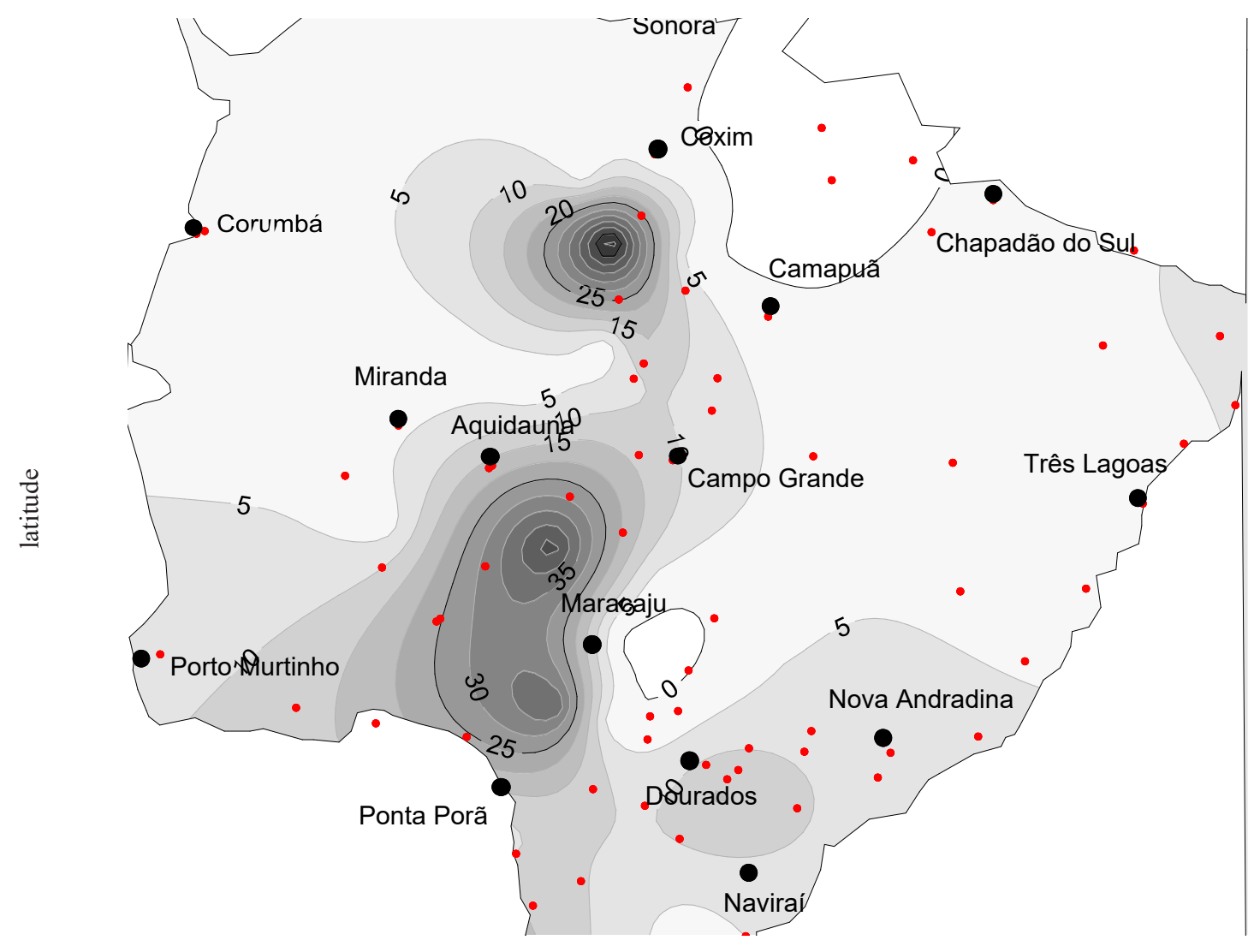

Longitude

*Pontos vermelhos indicam a localização da sede dos Municípios

Fonte: Centro Integrado de Vigilância Toxicológica (CIVITOX). Campo Grande, MS.

As regiões centro-norte, em torno de Rio Verde de Mato Grosso e Coxim, as intoxicações causadas por plantas ornamentais foram registradas entre a faixa etária de 20 e 58 anos e centro-sul, região de Dourados, entre 16 e 42 anos. Pesquisas relataram que em jovens e adultos (20-59 anos), as intoxicações causadas por plantas são menos frequentes. Essas intoxicações ocorrem principalmente devido ao contato acidental (BEYER et al., 2009; SIMÕES et al., 2017).

Já as demais regiões, com tonalidades mais claras, indicam que crianças e adolescentes sofreram algum tipo de intoxicação por esses vegetais, muitas vezes por não haver conhecimento sobre os riscos, bem como o acesso fácil a essas plantas.

De acordo com Tavares et al. (2013), a vulnerabilidade desta faixa etária consiste na oralidade das crianças que nos seus primeiros anos de vida tendem a introduzir objetos na boca. As crianças são seduzidas pela beleza e cores das plantas ornamentais, no entanto, quando tóxicas escondem riscos toxicológicos que variam desde uma simples coceira até o óbito.
Nota-se ainda, neste estudo um número expressivo de intoxicação com crianças de na faixa etária entre quatro a dez anos, esses dados são os mesmos descritos por Martins e Geron (2014), onde os autores comentam que, estatisticamente, a maior parte dos números de intoxicações se dá no interior das residências e as crianças são as principais vítimas, em função dessas espécies tóxicas serem bastante utilizadas em paisagismo residencial e ficarem ao alcance destas crianças.

O Centro de Informações Toxicológicas do Rio Grande do Sul (2014) disponibiliza dados que confirmam tal estatística. Em 2012, 43\% dos atendimentos referentes a intoxicações por plantas, envolviam crianças de até 05 anos e $15 \%$ crianças de 05 a 11 anos.

A análise das variáveis: ano, mês, idade, população e espécie baseada nos coeficientes de correlação linear de Pearson, estabelecendo o coeficiente de correlação entre as variáveis. Verifica-se que houve correlações significativas para ano e faixa etária com população e espécie e população com espécie, já a variável mês não teve correlação significativa com nenhuma outra variável (Quadro 2). 
Quadro 2 - nálise de correlação bivariada através dos coeficientes de correlação linear de Pearson. Campo Grande, MS

\begin{tabular}{|c|c|c|c|c|c|}
\hline \multicolumn{6}{|c|}{ Coeficiente de Correlação de Pearson $N=214$. Significância do teste (t) } \\
\hline & Ano & Mês & Idade & População & Espécie \\
\hline \multirow{2}{*}{ Ano } & \multirow{2}{*}{1.00000} & -0.05496 & -0.05280 & -0.13360 & 0.28585 \\
\hline & & 0.4238 & 0.4422 & 0.0510 & $<.0001$ \\
\hline \multirow{2}{*}{ Mês } & & \multirow{2}{*}{1.00000} & 0.04196 & 0.05734 & -0.02740 \\
\hline & & & 0.5415 & 0.4040 & 0.6902 \\
\hline \multirow{2}{*}{ Faixa Etária } & & & \multirow{2}{*}{1.00000} & $\mathbf{0 . 1 5 7 8 0}$ & 0.15012 \\
\hline & & & & 0.0209 & 0.0281 \\
\hline População & \multicolumn{3}{|c|}{0.0357} & 1.00000 & 0.14365 \\
\hline Espécie & & & & & 1.00000 \\
\hline & & & & & \\
\hline
\end{tabular}

Fonte: Dados da pesquisa.

$\mathrm{O}$ ano de 2006 apresentou maior número de casos de intoxicação (40 casos), levantados no CIVITOX, valor bem acima dos observados em relação a 2005 e entre 2007 a 2015. Desses 40 casos de intoxicação registrados em 2006, 35 foram registrados no município de Campo Grande, desse total, 16 foram ocasionados pela espécie comigo-ninguém-pode.

Quanto a variável faixa etária, os registros apontam que as crianças com dois anos de idade apresentaram maior número de intoxicação (39 casos), sendo a maior parte na região de Campo Grande, e a principal causa de intoxicações nessas crianças foi a espécie comigo-ninguém-pode. Essa informação valida a correlação entre faixa etária, população e espécie.

Em Campo Grande foram registrados, entre 2005 e 2015, 147 ocorrências por plantas ornamentais toxicas, de um total de 214. Dessas, 83 foram com a espécie comigo-ninguémpode e 20 com a coroa-de-cristo, comprovando a correlação entre população e espécie.

A Figura 4 é uma análise de componentes principais, análise multivariada, que correlaciona os principais componentes estudados: população (pop), idade (ida), ano estudado, mês do ano e espécie (esp) indicadas no caso de intoxicação. Os agrupamentos que as variáveis população, idade, mês do ano e espécies variam da mesma forma, ou seja, no decorrer dos meses do ano, os municípios com maiores populações foram mais acometidos por intoxicações por plantas ornamentais tóxicas, bem como pessoas mais velhas foram mais intoxicadas.

Figura 4 - Análise multivariada dos principais componentes levantados no CIVITOX em Mato Grosso do Sul, no período de 2005 a 2015. Campo Grande, MS

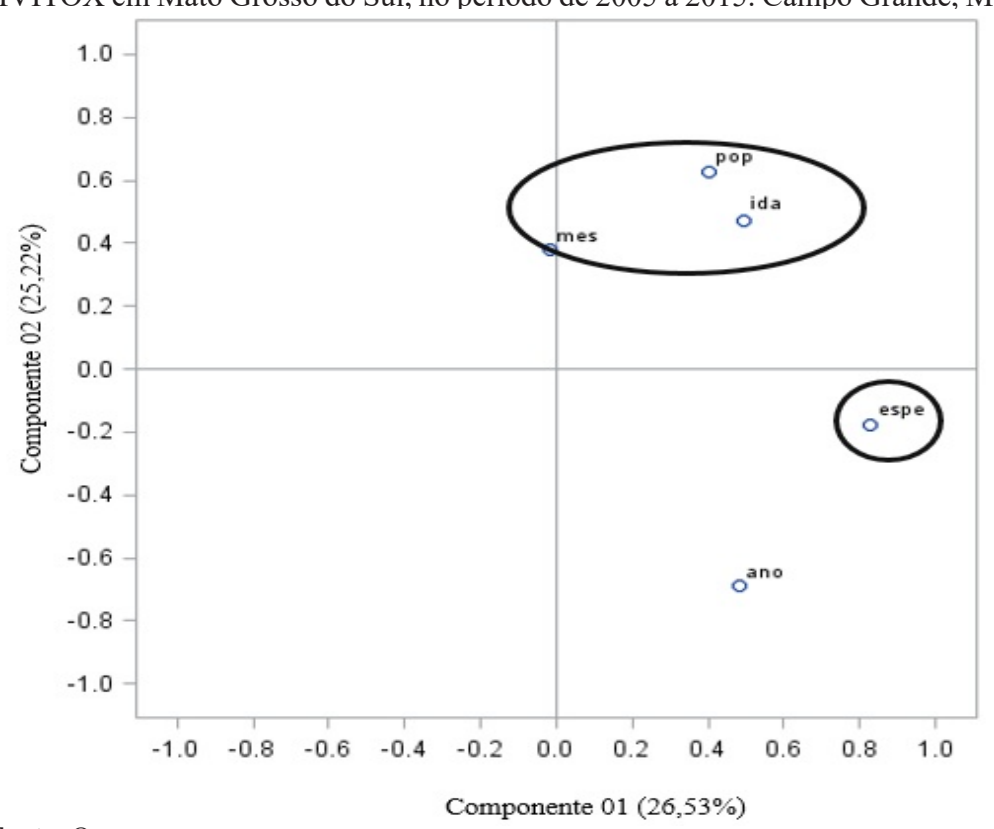

Fonte: Os autores.

A análise demonstra que população, idade e mês do ano ficaram mais agrupadas, indicando menor variabilidade entre elas, quando comparadas ao ano e a espécie. Este fato indica que as intoxicações estão mais relacionadas com as maiores populações, bem como pessoas mais velhas. No que diz respeito a espécie, esta varia de forma diferente, independe da idade, população e mês, o que significa que a espécie causadora da intoxicação nada tem a ver com as demais 
variáveis, ou seja, foram aleatórias quanto à espécie.

Em se tratando das pessoas mais velhas liderarem o grupo de intoxicações, cabe ressaltar que o cenário do Estado de Mato Grosso do Sul com uma rica biodiversidade, favorece o contato direto da população com a natureza. Além disso, os casos de intoxicação podem ser atribuídos a condição de saúde mais frágil da pessoa idosa em relação as mais jovens. Neste sentido, medidas preventivas poderiam ser adotadas, como a identificação de plantas com potencial de toxidez em praças e jardins.

\section{Conclusão}

A região central de Mato Grosso do Sul concentrou o maior número de ocorrências de intoxicação por plantas ornamentais tóxicas; já os índices de intoxicação por 5.000 habitantes mais elevados encontram-se na região de Rio Verde de Mato Grosso e Coxim e cerca de cinquenta casos de intoxicações por plantas ornamentais tóxicas com faixa etária mais elevada foi registrada nas regiões centro-norte e centro-sul de Mato Grosso do Sul e há correlação entre mês, população e idade de intoxicação.

A espécie predominante nos casos de intoxicação foi Dieffenbachia picta, num total de 135 casos no período em estudo, pertencente à família Araceae com um total de 150 casos. Os resultados sugerem que a escolha de plantas ornamentais para compor as áreas verdes dos municípios deve ser feita com critérios para minimizar os riscos para a população que utilizam esses espaços para o lazer.

\section{Agradecimentos}

A pesquisa trabalho foi realizada com apoio da Coordenação de Aperfeiçoamento de Pessoal de Nível Superior Brasil (CAPES) - Código de Financiamento 001 e, da Universidade Anhanguera-Uniderp. Ao CNPq, INAU, CPP, FUNDECT e FUNADESP, pelo apoio financeiro e a Bolsa de Produtividade CNPq-2.

\section{Referências}

ANDRADE FILHO, A.; CAMPOLINA, D.; DIAS, M. B. Toxicologia na prática clínica. Belo Horizonte: Folium, 2013.

AZEVEDO, J. L. S. A importância dos centros de informação e assistência toxicológica e sua contribuição na minimização dos agravos à saúde e ao meio ambiente no Brasil. Brasília: Universidade de Brasília, 2010.

BEYER, J. et al. Analysis of toxic alkaloids in body samples. Foren. Scien. Internat., v.185, p.1-9, 2009. doi: 10.1016/j. forsciint.2008.12.006.

BIONDI, D.; LEAL, L.; SCHAFFER, M. Aspectos importantes das plantas ornamentais em escolas públicas estaduais da cidade de Curitiba, PR. Rev. Bras. de Ciênc. Agrár., v.3, n.3, p.267-275, 2008. doi:10.5039/agraria.v3i3a325.

BURDA, A. M.; BURDA, N. M. The nation's first poison control center: taking a stand against accidental childhood poisoning in Chicago. Veter. Hum. Tox., v.39, n.2, p.115-119, 1997.

CIT (Centro Informações Toxicológicas - Rio Grande do Sul).
Estatística Dinâmica: intoxicações por faixa etária em 2012. Rio Grande do Sul, 2014. Disponível em: http://www.cit.rs.gov.br/ index.php?option $=$ com_wrapper\&view $=$ wrapper\&itemid $=60$. Acesso em 9 fev. 2020.

CUMPSTON, K. L. et al. Acute airway compromise after brief exposure to a Dieffenbachia plant. J. Emerg. Med., v.25, n.4, p.391-397, 2003. doi: 10.1016/j.jemermed.2003.02.005

DOMINATO, A. A. G. Levantamento estatístico das intoxicações exógenas relatadas por indivíduos atendidos em uma UBS do município de Presidente Prudente (SP). Colloq. Vit., v.4, n. especial, p. 31-38, 2012. doi: 10.5747/cv.2016.v08.nesp.000287

DUONG, T. H. et al. Further terpenoids from Euphorbia tirucalli. Fitoterapia, v. 135, p. 44-51, 2019. doi: 10.1016/j. fitote.2019.04.001.

FERNANDES, D. A. et al. Ethnobotanical survey of plants with toxic active constituents, grown in the municipality of Cuite, Paraíba, Brazil. Infarma-Ciênc. Farm., v.29, n.4, p.339-348, 2017. doi: 10.14450/2318-9312.

FIOCRUZ. Sistema nacional de informações tóxicofarmacológicas da Fundação Oswaldo Cruz (SINITOX), 2016. Disponível em:

<http://www.fiocruz.br/sinitox/cgi/cgilua.exe/sys/start. htm?sid=413>. Acesso em: mar. 2020.

GARCIA, R.M.S.; BALTAR, S.L.S.M.A. Registro e diagnóstico das intoxicações por plantas na cidade de Londrina (PR). Rev. Bras. Biociê., v.5, p.901-902, 2007.

IBGE. Censo de 2010. Disponível em: <http://www.ibge.gov. $\mathrm{br} /$ home/estatistica/populacao/censo2010/default.shtm $>$. Acesso em: 11 mar. 2020.

LORENZI, H. Plantas para Jardim no Brasil: herbáceas, arbustivas e trepadeiras. Nova Odessa: Instituto Plantarum, 2013..

MARTINS, T.D.; GERON, V.L. Plantas ornamentais tóxicas: conhecer para prevenir acidentes domésticos. Rev. Cient. FAEMA, v. 5, n.1, p. 79-98, 2014. doi: 10.31072/rcf.v5i1.222.

MATOS, F.J.A. et al. Plantas tóxicas: estudo de fitotoxicologia química de plantas brasileiras. São Paulo: Instituto Plantarum de Estudo da Flora, 2011.

MATOS, F.J.A. Plantas medicinais: guia de seleção e emprego de plantas usadas em fitoterapia no nordeste do Brasil. Fortaleza: UFC, 2000.

MENDIETA, M. C et al. Toxic plants: importance of knowledge for realization of health education. J. Nurs. UFPE, v.8, n.3, p.680686, 2014. doi: 10.5205/reuol.5149-42141-1-SM.0803201424.

SAS INSTITUTE. SAS Stat user's guide. Version 9. Carry: SAS Institute, 2002. 1 CDROM

SILVA, A.L.V.R.; USHIROBIRA, T.M.A. Aspectos toxicológicos da planta "comigo-ninguém-pode" (Dieffenbachia sp.). Rev UNINGÁ Review, v.2, p.64-69, 2010.

SILVA, P.H. et al. Entre a beleza e o perigo: uma abordagem sobre as plantas tóxicas ornamentais. Rev. Intertox-EcoAdvisor Tox. Ris. Amb. Soc., v.8, n.1, p.19-44, 2015.doi: 10.22280/ revintervol8ed1.192.

SIMÕES, C.M.O. et al. Farmacognosia: do produto natural ao medicamento. Florianopolis: UFSC, 2017.

SURFER, version 8.0. Apostila do Curso Básico de Surfer - versão 8.0. Surface Mapping System Copyright $@$, Golden Software, Inc. (informações em http://www.goldensoftware.com).

TAVARES, E.O. et al. Fatores associados à intoxicação infantil. Escola Anna Nery. Rev. Enferm. v.17, n.1, p.31-37, 2013. 
doi.10.1590/S1414-81452013000100005.

TOKARNIA, C.H.; DOBEREINER, J.; PEIXOTO, P.V. Plantas tóxicas do Brasil. Rio de Janeiro: Helianthus, 2000.

UEMURA, D.; HIRATA, Y. Farmacognosia: da planta ao medicamento. Porto Alegre: UFRGS, 2001.
VASCONCELOS, J.; VIEIRA, J.G.P.; VIEIRA, E.P.P. Plantas tóxicas: conhecer para prevenir. Rev. Cient. UFPA, v.7, n.1, p.1$10,2009$.

WINTERS, G. Plantas ornamentais tóxicas. Holambra: Centro Paisagístico Gustaaf Winters, 2013. 